\title{
Combined expression of ezrin and E-cadherin is associated with lymph node metastasis and poor prognosis in breast cancer
}

\author{
ZHAOJIN YU ${ }^{1}$, MINGLI SUN ${ }^{1}$, FENG JIN $^{3}$, QINGHUAN XIAO ${ }^{2}$, MIAO HE $^{1}$, HUIZHE WU $^{1}$, JIE REN $^{1}$, LIN ZHAO $^{1}$, \\ HAISHAN ZHAO $^{1}$, WEIFAN YAO ${ }^{1}$, FENGPING SHAN ${ }^{4}$, YAMING CAO $^{4}$ and MINJIE WEI ${ }^{1}$ \\ ${ }^{1}$ Department of Pharmacology, ${ }^{2}$ Deparment of Ion Channel Pharmacology, School of Pharmacy, China Medical University, \\ Shenyang, Liaoning 110122; ${ }^{3}$ Department of Breast Surgery, The First Hospital of China Medical University, \\ Shenyang, Liaoning 110001; ${ }^{4}$ Department of Immunology, School of Basic Medical Science, \\ China Medical University, Shenyang, Liaoning 110122, P.R. China
}

Received February 27, 2015; Accepted April 20, 2015

DOI: $10.3892 /$ or.2015.3967

\begin{abstract}
Ezrin and E-cadherin have been known to play a role in tumor metastasis. However, the association between the expression of ezrin and E-cadherin in breast cancer patients remains unclear. In the present study, we investigated the expression of ezrin and E-cadherin in 275 breast cancer and 80 control patients with benign hyperplasia, using tissue microarray-based immunohistochemistry (IHC). Ezrin expression was higher, while that of E-cadherin was lower in breast cancer than in control samples. Ezrin expression was negatively correlated with E-cadherin expression in a subpopulation of breast cancer patients with a high expression of ezrin [ezrin(high)] and a low expression of E-cadherin [E-cad(low)]. The cytoplasmic expression of E-cadherin [E-cad(c)] occurred significantly more frequently in ezrin(high) breast cancer than in ezrin(low) breast cancer. The expression level of ezrin was significantly higher in breast cancer with E-cad(c) than that with a membrane expression of E-cadherin [E-cad(m)]. Ezrin(high) or E-cad(low) expression was more associated with lymph node metastasis, and shorter overall survival (OS) and disease-free survival (DFS) in breast cancer patients. E-cad(c) was more associated with lymph node metastasis and shorter OS and DFS compared with E-cad(m). The combined expression of ezrin(high) and E-cad(low) or ezrin(high) and
\end{abstract}

Correspondence to: Professor Minjie Wei, Department of Pharmacology, School of Pharmacy, China Medical University, 77 Puhe Road, Shenyang North New Area, Shenyang, Liaoning 110122, P.R. China

E-mail:weiminjiecmu@163.com

Abbreviations: AUCs, areas under curves; CI, confidence interval; DAB, 3,3'-diaminobenzidine; DFS, disease-free survival; E-cad, E-cadherin; EMT, epithelial-mesenchymal transition; OS, overall survival; ROC, receiver operating characteristic; RR, relative risk; TGF- $\beta 1$, transforming growth factor- $\beta 1$; TNM, tumor-nodemetastasis; TMA, tissue microarray

Key words: breast cancer, ezrin, E-cadherin, metastasis ezrin(c) was more associated with lymph node metastasis and poor prognosis. In addition, the multivariate Cox regression analysis revealed that lymph node metastasis and ezrin(high) expression were independent prognostic factors for shorter OS and DFS in breast cancer patients. The results of the present study suggested that ezrin promotes breast cancer metastasis via the regulation of E-cadherin expression.

\section{Introduction}

Breast cancer is the most common malignant disease in women, and is one of the leading causes of cancer-related mortality worldwide. Although it is not the primary tumor, its distant metastasis is the main cause of breast cancer-related mortality (1). It is estimated that $\sim 20-30 \%$ of breast cancer patients develop metastasis (2). Chemotherapy with taxanes or combination therapy with trastuzumab has been reported to improve the survival rate in patients with metastatic breast cancer (2). Therefore, identification of prognostic markers of metastasis risk can identify breast cancer patients with a high risk for developing metastasis, and thus can help to improve, the clinical management of these patients.

Tumor metastasis involves multiple processes such as cell invasion into the basal membrane, spread into the lymph node or blood vessel and growth in distant tissues. At each process, tumor cells undergo morphological changes that require dynamic modeling of the actin cytoskeleton (3). Ezrin, belonging to the ezrin, radixin and moesin protein family, acts as a connector between the actin cytoskeleton and plasma membrane, and regulates cell-cell and cell-matrix adhesion (4). Evidence has shown that ezrin plays an important role in tumor cell invasion and metastasis (5-7). It has been reported that ezrin overexpression is associated with metastasis in numerous malignant tumors such as osteosarcoma, pancreatic cancer, hepatocellular and tongue squamous cell carcinoma, and non-small-cell lung cancer (8-11). Ezrin overexpression has been found to be associated with lymph node metastasis in breast cancer patients $(12,13)$. The role of ezrin in breast cancer cell metastasis is also supported by several studies showing that ezrin overexpression promotes lung metastasis and ezrin silencing reverses breast cancer cell metastasis $(14,15)$. 
However, the mechanisms by which ezrin triggers breast cancer cell metastasis remain poorly understood.

Epithelial-mesenchymal transition (EMT), a process in which an immobile epithelial cell changes into a mobile mesenchymal cell, has been known to play an important role in cancer cell metastasis (16). Loss of E-cadherin (E-cad), a hallmark of EMT, has been found in tumors such as oral squamous cell carcinoma, ovarian cancer, colorectal and hepatocellular carcinoma, and breast cancer, and is associated with tumor cell invasion and metastasis (17-23). It has been reported that knockdown of ezrin increases the expression of E-cad in breast cancer and tongue squamous cell carcinoma cell lines $(11,15)$. In addition, ezrin has been reported to be required for TGF- $\beta 1$-induced EMT in lung cancer cells (24). However, the association between the expression of ezrin and E-cad in breast cancer patients remains unclear.

In the present study, we investigated the expression of ezrin and E-cad in 275 breast cancer and 80 control patients with benign hyperplasia. The purpose of the present study was to analyze the association between the expression of ezrin and E-cad in breast cancer patients, and to examine the correlation of their expression with clinicopathological characteristics and prognosis of breast cancer patients.

\section{Materials and methods}

Patients and tissue samples. The Medical Ethics Committee of the China Medical University approved the present study. Due to the retrospective nature of the study, the Ethics Committee waived the need for written informed consent by the patients.

Human breast tissues were obtained from 275 female patients with breast cancer and 80 control patients with benign hyperplasia of mammary glands, who underwent surgery at the First Affiliated Hospital of China Medical University between 2004 and 2008. The average age of patients with breast cancer was 50.9 \pm 9.6 years (range, 29-79 years). The diagnosis of breast cancer and benign hyperplasia was confirmed by pathological staining. Of the 275 breast cancer patients, 219 patients had invasive ductal carcinoma, 30 patients had invasive lobular carcinoma and 26 patients had other types of tumors including mucinous, medullary and cribriform carcinoma. The stage of the cancer was evaluated in 198 patients according to the TNM staging system as follows: stage I $(n=46)$, stage II $(n=122)$ and stage III $(n=30)$. The histological grading of the cancer was performed in 265 patients according to the World Health Organization grading system as follows: grade $I(n=136)$ and grade II-III ( $n=129)$. Clinicopathological data including patient age, menopausal status, tumor size and lymph node metastasis were retrospectively retrieved from medical records. The patients did not undergo radiation therapy and chemotherapy prior to surgery.

Eighty patients with benign hyperplasia of mammary glands were used as controls. The average age of patients with benign hyperplasia of mammary glands was $50.2 \pm 10.2$ years (range, 23-72 years).

Tissue microarray (TMA) and immunohistochemistry (IHC). Paraffin blocks (donor blocks) containing representative tumor and hyperplasia samples were selected by reviewing the hematoxylin and eosin-stained slides. Tissue cores with a diameter of $1.5 \mathrm{~mm}$ were extracted from each donor block, and precisely arrayed into a new paraffin recipient block with a maximum of 200 cores, using the Organization Microarrayer (Pathology Devices, Westminster, MD, USA). Sections (4- $\mu \mathrm{m})$ were obtained from formalin-fixed and paraffin-embedded TMA blocks, mounted on poly-L-lysine-coated glass slides and used for IHC.

The sections were deparaffinized with xylene, rehydrated in a graded alcohol series and heated in a microwave oven to retrieve antigen. Endogenous peroxidase activity was blocked with $3 \% \mathrm{H}_{2} \mathrm{O}_{2}$ at $37^{\circ} \mathrm{C}$ for $20 \mathrm{~min}$. The sections were incubated with primary antibodies against ezrin (ab4069, mouse anti-human monoclonal antibodies; 1:100 dilution) and E-cadherin (ab1416, mouse anti-human monoclonal antibodies; 1:100 dilution) (both from Abcam Plc, Cambridge, $\mathrm{UK}$ ) overnight at $4^{\circ} \mathrm{C}$. After the primary antibodies were washed off, the sections were incubated with biotinylated secondary antibody for $30 \mathrm{~min}$ at $37^{\circ} \mathrm{C}$. The sections were then incubated with streptavidin horseradish peroxidase for an additional 30 min (LSAB kit; Dako, Glostrup, Denmark), and stained with 3,3'-diaminobenzidine (DAB). The sections were counterstained with hematoxylin, dehydrated and mounted. Any sections in which primary antibodies were omitted were used as negative controls.

Evaluation of IHC. Immunostaining was examined under a light microscope by two pathologists who were blinded to the experimental conditions. The intensity of immunoreactivity was scored as follows: 0 , for no staining; 1 , for weak staining; 2, for moderate staining; and 3, for strong staining. The percentage of stained cells was scored as $0-100 \%$. The final immunoreactive score was determined by multiplying the intensity score with the percentage of positively stained cells. The minimum score was 0 and the maximum score was $300 \%$. Scores were assigned using $5 \%$ increments $(0,5,10, \ldots .300 \%)$, as previously reported $(25,26)$. The scores for each sample were used to determine the cut-off value for discriminating tumors with a high expression of ezrin or E-cadherin from tumors with a low expression, using receiver operating characteristic (ROC) curves. To discriminate tumors with the cytoplasmic expression of E-cad from tumors with the membrane expression of E-cad, the percentage of E-cad membrane expression for each sample was used to determine the cut-off value, using ROC curves. The percentage of E-cad membrane expression was calculated as follows: E-cad membrane expression $\%=$ the number of cells with membrane expression/(the number of cells with membrane expression + the number of cells with cytoplasmic expression) $\mathrm{x} 100 \%$. The sensitivity and specificity for the survival status (alive or dead) of breast cancer patients was plotted to generate ROC curves.

Statistical analysis. Data are presented as mean \pm SD. Statistical analyses were performed using SPSS 11.5 (Chicago, IL, USA). For data with normal distribution, the Student's t-test was used to compare the difference in the means between groups. For data with unequal variance, the Wilcoxon rank-sum or Kruskal-Wallis tests were used to compare the difference. The Pearson's Chi-square or Fisher's exact probability tests were used to evaluate the association between the expression of ezrin and E-cad and clinicopathological characteristics of 


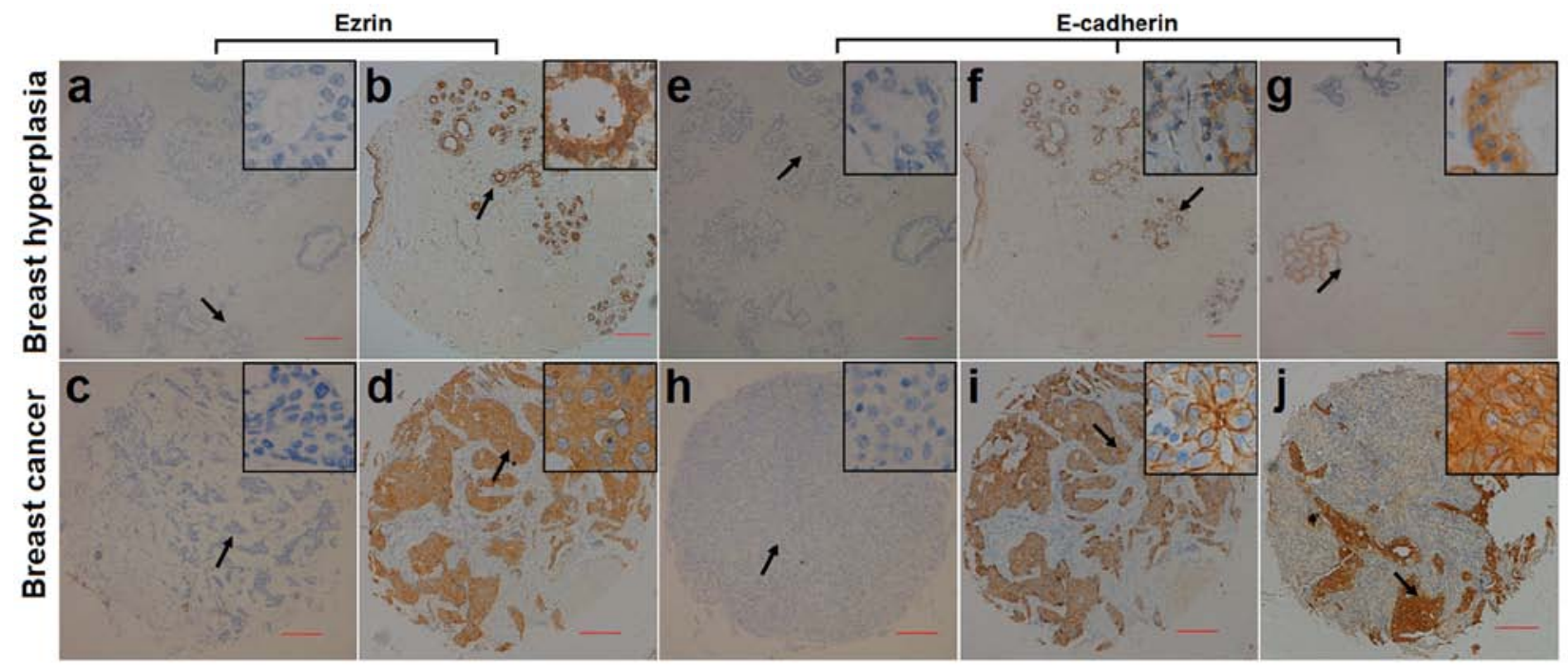

Figure 1. Representative micrographs showing negative (a, c, e and h) and positive (b, d, f, g, i and j) immunohistochemical staining of (a-d) ezrin and (e-j) E-cadherin in breast hyperplasia (a, b, e, f and g) and breast cancer (c, d, h, i and j). Magnification, $x 40$. Arrows shows the magnified regions in the insert (x400). Scale bar, $200 \mu \mathrm{m}$.

breast cancer patients. The Spearman's and Pearson's rank correlation analyses were applied to assess the association between the expression of ezrin and E-cad. Survival probabilities were estimated by the Kaplan-Meier method and assessed by a log-rank test. The univariate and multivariate Cox proportional hazards regression models were used to assess the association between potential confounding variables and prognosis (OS or DFS). The OS was calculated as the time between the first day of diagnosis and disease-related death or last known follow-up. The DFS was calculated as the time between the first day of diagnosis and the occurrence of local recurrence or distant metastasis. Probability values $\leq 0.05$ were considered to indicate a statistically significant result.

\section{Results}

Chinicopathological characteristics of patients with breast cancer. Table I shows the clinicopathological characteristics of 275 patients with breast cancer. The age, menopausal status, tumor size, tumor type, histological grade, TNM stage and lymph node metastasis were recorded in 275, 266, 228, 275, 265, 198 and 265 patients, respectively. The majority of these patients had a tumor with invasive ductal carcinoma (79.6\%), $\leq 2 \mathrm{~cm}$ in size $(62.3 \%)$, histological grade II-III $(51.3 \%)$ and TNM stage II (61.6\%). Lymph node metastasis occurred in 94 (25.9\%) of 265 patients.

Follow-up information was available for 169 breast cancer patients. During the follow-up period of 9-118 months, relapses occurred in 62 cases and cancer-associated deaths were found in 48 cases. The 5 -year survival rate was $84.6 \%$. The mean OS and DFS were 98.5 and 91.3 months, respectively.

Expression of ezrin and E-cad in breast cancer. The expression of ezrin and E-cad was examined in 275 samples from breast cancer patients and 80 control samples from patients with benign hyperplasia, using IHC (Fig. 1). Ezrin-immunopositive staining was mainly observed in the cytoplasm in the breast cancer and control samples. Ezrin-immunopositive staining
Table I. Clinicopathological characteristics of breast cancer patients.

\begin{tabular}{|c|c|c|}
\hline \multirow[b]{2}{*}{ Characteristics } & \multicolumn{2}{|c|}{ Cases } \\
\hline & $\mathrm{n}$ & $(\%)$ \\
\hline Age (years) & 275 & \\
\hline$\leq 50$ & 145 & 52.7 \\
\hline$>50$ & 130 & 47.3 \\
\hline Menopausal status & 266 & \\
\hline Pre-menopause & 148 & 55.6 \\
\hline Post-menopause & 118 & 44.4 \\
\hline Tumor size (cm) & 228 & \\
\hline$\leq 2.0$ & 142 & 62.3 \\
\hline$>2.0$ & 86 & 37.7 \\
\hline Tumor type & 275 & \\
\hline Ductal & 219 & 79.6 \\
\hline Lobular & 30 & 10.9 \\
\hline Others & 26 & 9.5 \\
\hline Histological grade & 265 & \\
\hline I & 136 & 51.3 \\
\hline II-III & 129 & 48.7 \\
\hline TNM stage & 198 & \\
\hline I & 46 & 23.2 \\
\hline II & 122 & 61.6 \\
\hline III & 30 & 15.2 \\
\hline Lymph node metastasis & 265 & \\
\hline No & 171 & 64.5 \\
\hline Yes & 94 & 35.5 \\
\hline
\end{tabular}

was observed in 239 (86.9\%) of 275 breast cancer samples and $61(76.2 \%)$ of 80 control samples. Ezrin immunoreactivity 
a

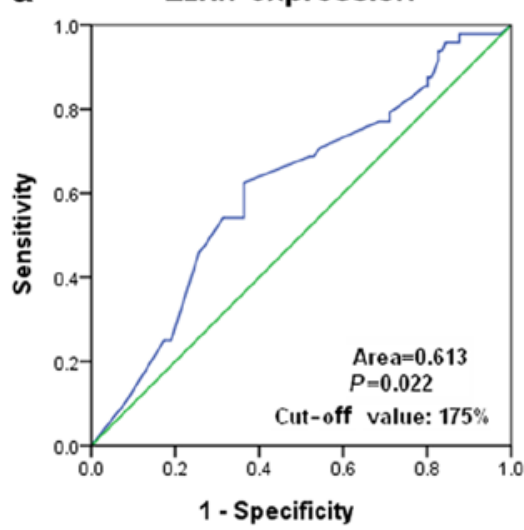

b

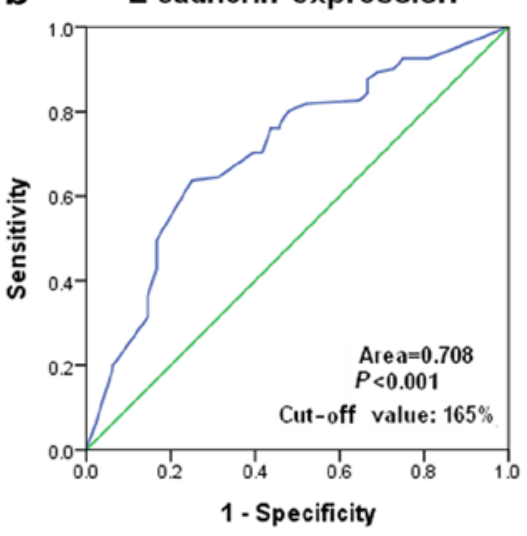

C

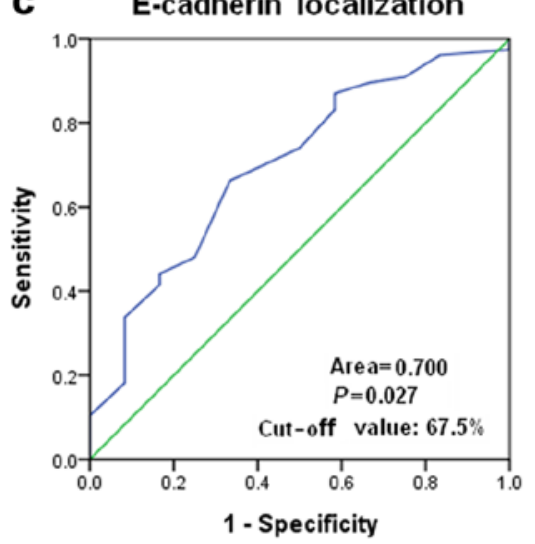

Figure 2. Receiver operating characteristic curves were used to determine the cut-off value for the expression of (a) ezrin and (b) E-cadherin, and subcellular expression of E-cadherin (c) based on the survival status of breast cancer patients. The sensitivity and specificity for each outcome were plotted and the areas under curve (AUCs) and p-value were indicated.

Table II. Correlation between the expression of ezrin and E-cadherin in breast cancer.

\begin{tabular}{|c|c|c|c|c|c|c|}
\hline \multirow[b]{2}{*}{ Expression } & \multicolumn{3}{|c|}{ E-cad(low) } & \multicolumn{3}{|c|}{ E-cad(high) } \\
\hline & $\mathrm{n}$ & $\mathrm{r}^{\mathrm{a}}$ & $\mathrm{P}^{\mathrm{b}}$ & $\mathrm{n}$ & $\mathrm{r}^{\mathrm{a}}$ & $\mathrm{P}^{\mathrm{b}}$ \\
\hline Ezrin(low) & 68 & -0.101 & 0.411 & 83 & -0.062 & 0.579 \\
\hline Ezrin(high) & 63 & -0.286 & 0.023 & 61 & -0.082 & 0.531 \\
\hline
\end{tabular}

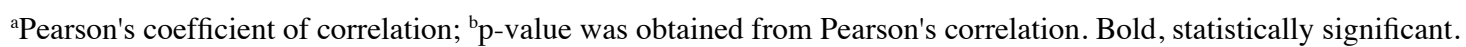

occurred significantly more frequently in breast cancer samples than in control samples $(\mathrm{p}=0.020)$. By contrast, E-cad immunoreactivity was observed in the membrane and cytoplasm in breast cancer and control samples. E-cadimmunopositive staining was observed in $205(74.5 \%)$ of 275 breast cancer samples and $70(87.5 \%)$ of 80 control samples. E-cad immunoreactivity occurred significantly less frequently in breast cancer samples than in control samples $(\mathrm{p}=0.015)$.

Selection of the cut-off value for the expression of ezrin and $E$-cad. A ROC curve analysis was performed to determine an optimal cut-off score for the expression of ezrin and E-cad in breast cancer samples. Based on the survival status, cut-off values of 175 and $165 \%$ were selected for the expression of ezrin and E-cad, respectively (Fig. 2a and b). Tumors with immunohistological scores $\geq 175$ and $<175 \%$ were defined as tumors with 'high' [ezrin(high)] and 'low' [ezrin(low)] expression of ezrin, respectively. Tumors with immunohistological scores $\geq 165$ and $<165 \%$ were defined as tumors with 'high' [E-cad(high)] and 'low' (E-cad(low) expression of E-cad, respectively. Tumors 124 (45.1\%) exhibited an ezrin(high) expression and 151 (54.9\%) tumors showed an ezrin(low) expression. Tumors 144 (52.4\%) exhibited E-cad(high) expression and 131 (47.6\%) tumors showed E-cad(low) expression.

We also performed the ROC analysis to determine an optimal cut-off score for discriminating the membrane expression from the cytoplasmic expression of E-cad in breast cancer with an E-cad(high) expression. Based on the survival status, a cut-off score of $67.5 \%$ was selected (Fig. 2c). Tumors with membrane expression of $\geq 67.5$ and $<67.5 \%$ were defined as tumors with E-cad membrane [E-cad(m)] and E-cad cytoplasmic [E-cad(c)] expression, respectively. Of the 144 E-cad(high) breast cancer, 85 (59.0\%) tumors had E-cad(m) expression and 59 (41.0\%) tumors had E-cad(c) expression.

Association between the expression of ezrin and E-cad in breast cancer. We investigated the association between the expression of ezrin and E-cad in breast cancer, using Spearman's rank correlation analysis. There was no significant correlation between the expression of ezrin and E-cad in breast cancer patients $(n=275)$. We also investigated the association between the expression of ezrin and E-cad in subgroups of breast cancer with different expression levels of ezrin and E-cad. The Pearson's correlation analysis showed a negative correlation between the expression of ezrin and E-cad in breast cancer with an ezrin(high) and E-cad(low) expression $(r=-0.286, p=0.023$, Table II).

We then examined the subcellular expression levels of E-cad in breast cancer with E-cad(high). In breast cancer with ezrin(low) expression, E-cad(m) expression was observed in $56(67.5 \%)$ of 83 cases and E-cad(c) expression was found in $27(32.5 \%)$ of 83 cases. In breast cancer with ezrin(high) expression, E-cad(m) expression was observed in $29(47.5 \%)$ of 61 cases, and E-cad(c) expression was found in $32(52.5 \%)$ of 61 cases. E-cad(c) expression occurred significantly more 
Table III. Association of ezrin and E-cadherin expression with clinicopathological features of breast cancer.

\begin{tabular}{|c|c|c|c|c|c|c|c|c|c|}
\hline \multirow[b]{2}{*}{ Characteristics } & \multicolumn{3}{|c|}{ Ezrin } & \multicolumn{3}{|c|}{ E-cadherin } & \multicolumn{3}{|c|}{ E-cadherin localization ${ }^{\mathrm{b}}$} \\
\hline & $\begin{array}{l}\text { High } \\
\mathrm{n}(\%)\end{array}$ & $\begin{array}{l}\text { Low } \\
\mathrm{n}(\%)\end{array}$ & $\mathrm{P}^{\mathrm{a}}$ & $\begin{array}{l}\text { High } \\
\mathrm{n}(\%)\end{array}$ & $\begin{array}{l}\text { Low } \\
\mathrm{n}(\%)\end{array}$ & $\mathrm{P}^{\mathrm{a}}$ & $\begin{array}{c}\text { E-cad(c) } \\
\mathrm{n}(\%)\end{array}$ & $\begin{array}{c}\text { E-cad(m) } \\
\mathrm{n}(\%)\end{array}$ & $\mathrm{P}^{\mathrm{a}}$ \\
\hline $\begin{array}{l}\text { Age (years) } \\
\text { at diagnosis }\end{array}$ & & & 0.191 & & & 0.325 & & & 0.198 \\
\hline$\leq 50$ & $60(41.4)$ & $85(58.6)$ & & $80(55.2)$ & $65(44.8)$ & & $29(36.2)$ & $51(63.8)$ & \\
\hline$>50$ & $64(49.2)$ & $66(50.8)$ & & $64(49.2)$ & $66(50.8)$ & & $30(46.9)$ & $34(53.10$ & \\
\hline Menopaused status & & & 0.022 & & & 0.131 & & & 0.093 \\
\hline Pre-menopause & $57(38.5)$ & $91(61.5)$ & & $84(56.8)$ & $64(43.2)$ & & $30(35.7)$ & $54(64.3)$ & \\
\hline Post-menopause & $62(52.5)$ & $56(47.5)$ & & $56(47.5)$ & $62(52.5)$ & & $28(50.0)$ & $28(50.0)$ & \\
\hline Tumor size $(\mathrm{cm})$ & & & 0.512 & & & 0.392 & & & 0.058 \\
\hline$\leq 2.0$ & $63(44.4)$ & $79(55.6)$ & & $76(53.5)$ & $66(46.5)$ & & $27(35.5)$ & $49(64.5)$ & \\
\hline$>2.0$ & $42(48.8)$ & $44(51.2)$ & & $41(47.7)$ & $45(52.3)$ & & $22(53.7)$ & $19(46.3)$ & \\
\hline Tumor type & & & 0.071 & & & 0.165 & & & 0.586 \\
\hline Ductal & $106(48.4)$ & $113(51.6)$ & & $115(52.5)$ & $104(47.5)$ & & $49(42.6)$ & $66(57.4)$ & \\
\hline Lobular & $11(36.7)$ & $19(63.3)$ & & $12(40.0)$ & $18(60.0)$ & & $5(41.7)$ & $7(58.3)$ & \\
\hline Others & 7 (26.9) & $19(73.1)$ & & $17(65.4)$ & 9 (34.6) & & $5(29.4)$ & $12(70.6)$ & \\
\hline Histological grade & & & 0.001 & & & 0.251 & & & 0.408 \\
\hline I & $47(34.6)$ & $89(65.4)$ & & $76(55.9)$ & $60(44.1)$ & & $33(43.4)$ & $43(56.6)$ & \\
\hline II-III & $71(55.0)$ & $58(45.0)$ & & $63(48.8)$ & $66(51.2)$ & & $23(36.5)$ & $40(63.5)$ & \\
\hline TNM stage & & & 0.197 & & & 0.007 & & & 0.233 \\
\hline I & $16(34.8)$ & $30(65.2)$ & & $28(60.9)$ & $18(39.1)$ & & $12(42.9)$ & $16(57.1)$ & \\
\hline II & $59(48.4)$ & $63(51.6)$ & & $68(55.7)$ & $54(44.3)$ & & $30(44.1)$ & $38(55.9)$ & \\
\hline III & $16(53.3)$ & $14(46.7)$ & & $8(26.7)$ & $22(73.3)$ & & $6(75.0)$ & $2(25.0)$ & \\
\hline $\begin{array}{l}\text { Lymph node } \\
\text { metastasis }\end{array}$ & & & 0.002 & & & $<0.001$ & & & 0.047 \\
\hline No & $66(38.6)$ & $105(61.4)$ & & $104(60.8)$ & $67(39.2)$ & & $38(36.5)$ & $66(63.5)$ & \\
\hline Yes & $55(58.5)$ & 39 (41.5) & & $34(36.2)$ & $60(63.8)$ & & $19(55.9)$ & $15(44.1)$ & \\
\hline
\end{tabular}

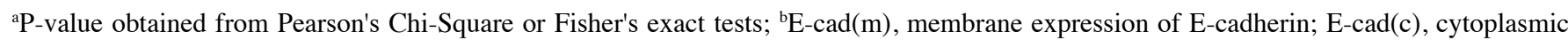
expression of E-cadherin. Bold, statistically significant.

frequently in ezrin(high) breast cancer than in ezrin(low) breast cancer (Chi-square, $p=0.016$, Fig. 3a). Furthermore, the expression level of ezrin was significantly higher in E-cad(c) breast cancer than in $E$-cad $(m)$ breast cancer $(p=0.029$, Fig. 3b).

Association of the expression of ezrin and E-cad with clinico pathological characteristics of breast cancer patients. We investigated the association between the expression of ezrin and E-cad and the clinicopathological characteristics of breast cancer patients (Table III). The age, tumor size and TNM stage were not significantly associated with the expression of ezrin. Ezrin(high) expression was more associated with postmenopausal status ( $\mathrm{p}=0.022)$, histological grade II-III $(\mathrm{p}=0.001)$ and lymph node metastasis ( $\mathrm{p}=0.002$ ). The age, menopausal status, tumor size, tumor type and histological grade were not significantly associated with the expression of E-cad. E-cad(low) expression was more associated with TNM stage II $(p=0.007)$ and lymph node metastasis $(\mathrm{p}<0.001)$. Furthermore, E-cad(c) expression was more associated with lymph node metastasis $(\mathrm{p}=0.047)$.

Since ezrin(high), E-cad(low) and E-cad(c) expression was associated with lymph node metastasis, we further investigated the association of their combined expression with lymph node metastasis (Table IV). Compared with tumors with ezrin(low) and E-cad(high) expression, ezrin(low) and E-cad(low) expression or ezrin(high) and E-cad(high) expression, tumors with ezrin(high) and E-cad(low) expression were more associated with lymph node metastasis ( $p<0.05$, Table IV). Compared with tumors with ezrin(low) and E-cad(high) expression, tumors with ezrin(low) and E-cad(low) expression or ezrin(high) and E-cad(high) expression was more associated with lymph node metastasis ( $<<0.05$, Table IV). In addition, compared with tumors with ezrin(low) and E-cad(m) expression, tumors with ezrin(low) and E-cad(c) expression, ezrin(high) and E-cad(m) expression or ezrin(high) and E-cad(c) expression were more associated with lymph node metastasis (Table IV). 

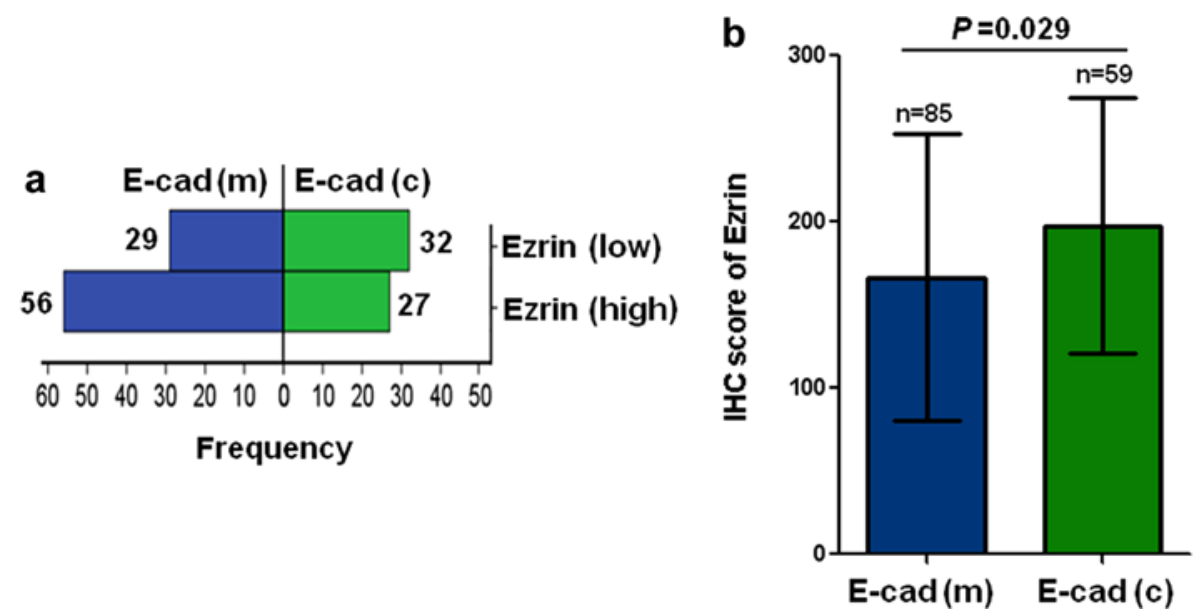

Figure 3. The association between the subcellular expression of E-cad and ezrin expression in breast cancer. (a) E-cad(m) and E-cad(c) expression in breast cancer with ezrin(high) and ezrin(low) expression. (b) The IHC scores of ezrin in breast cancer with E-cad(m) and E-cad(c) expression. P=0.029. IHC, immunohistochemistry.

Table IV. Association of combination of ezrin and E-cadherin with lymph-node metastasis of breast cancer.

\begin{tabular}{|c|c|c|c|c|c|}
\hline & \multicolumn{2}{|c|}{ Lymph-node metastasis } & \multirow[b]{2}{*}{$\mathrm{P}^{\mathrm{g}}$} & & \\
\hline & No $(\%)$ & Yes $(\%)$ & & & \\
\hline Combination of ezrin and E-cadherin & & & $<0.001$ & & \\
\hline Ezrin(low) + E-cad(high) & $64(82.1)$ & $14(17.9)$ & & & \\
\hline Ezrin(low) + E-cad(low) & $40(66.7)$ & $20(33.3)$ & $\mathbf{0 . 0 3 8}^{\mathrm{a}}$ & & \\
\hline Ezrin(high) + E-cad(high) & $41(62.1)$ & $25(37.9)$ & $\mathbf{0 . 0 0 7}^{\mathrm{a}}$ & $0.595^{\mathrm{b}}$ & \\
\hline Ezrin(high) + E-cad(low) & $26(42.6)$ & $35(57.4)$ & $<0.001^{\text {a }}$ & $0.008^{b}$ & $\mathbf{0 . 0 2 8}{ }^{\mathrm{c}}$ \\
\hline Combination of ezrin and E-cadherin localization ${ }^{\mathrm{h}}$ & & & 0.041 & & \\
\hline Ezrin $($ low $)+$ E-cad $(m)$ & $47(88.7)$ & $6(11.3)$ & & & \\
\hline Ezrin $($ low $)+$ E-cad(c) & $17(68.0)$ & $8(32.0)$ & $\mathbf{0 . 0 5 4} 4^{\mathrm{d}}$ & & \\
\hline Ezrin(high) + E-cad(m) & $19(67.9)$ & $9(32.1)$ & $\mathbf{0 . 0 2 2}{ }^{\mathrm{d}}$ & $0.991^{\mathrm{e}}$ & \\
\hline Ezrin(high $)+$ E-cad(c) & $21(65.6)$ & $11(34.4)$ & $0.010^{d}$ & $0.850^{\mathrm{e}}$ & $0.855^{\mathrm{f}}$ \\
\hline
\end{tabular}

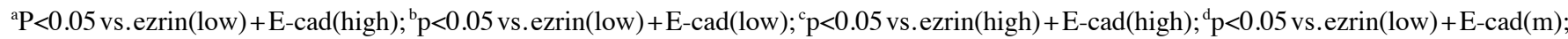
${ }^{\mathrm{e}} \mathrm{p}<0.05$ vs. ezrin(low $+\mathrm{E}-\mathrm{cad}(\mathrm{c}) ;{ }^{\mathrm{f}} \mathrm{p}<0.05$ vs. ezrin(high) $+\mathrm{E}-\mathrm{cad}(\mathrm{m}) .{ }^{\mathrm{g}} \mathrm{P}$-value obtained from Pearson's Chi-Square or Fisher's exact tests; ${ }^{\mathrm{h}} \mathrm{E}-$ $\operatorname{cad}(\mathrm{m})$, membrane expression of E-cadherin; E-cad(c), cytoplasmic expression of E-cadherin. Bold, statistically significant.

Association of the expression of ezrin and E-cad with the survival of breast cancer patients. We evaluated the association of the expression level of ezrin and E-cad with the OS or DFS in breast cancer patients, using the Kaplan-Meier analysis and log-rank test. Ezrin(high) expression was associated with shorter OS ( $p=0.002$, Fig. $4 a)$ and DFS ( $p=0.010$, Fig. 4b). E-cad(low) expression was associated with shorter OS $(\mathrm{p}<0.001$, Fig. 4c) and DFS ( $<<0.001$, Fig. 4d). In addition, E-cad(c) expression was associated with a significantly shorter OS ( $p=0.030$, Fig. 4e). Although E-cad(c) expression exhibited a tendency towards a shorter DFS, no statistically significant difference was found ( $\mathrm{p}=0.137$, Fig. 4f).

We also examined the association of the combined expression of ezrin and E-cad with the OS or DFS in breast cancer patients. Compared with tumors with ezrin(low) and E-cad(high) expression, tumors with ezrin(low) andE-cad(low) expression, ezrin(high) and E-cad(high) expression, and ezrin(high) and E-cad(low) expression were associated with shorter OS and DFS in breast cancer patients (Fig. 5a and b). Compared with ezrin(low) and E-cad(low) expression and ezrin(high) and E-cad(high) expression, ezrin(high) and E-cad(low) expression was associated with shorter OS and DFS in breast cancer patients (Fig. 5a and b). In addition, compared with tumors with ezrin(low) and E-cad(m) expression, tumors with ezrin(low) and E-cad(c) expression, ezrin(high) and E-cad(m) expression, and ezrin(high) and E-cad(c) expression were associated with shorter OS and DFS in breast cancer patients (Fig. 5c and d).

A univariate Cox regression model was used to estimate the impact of each clinicopathological variable on the OS and DFS in breast cancer patients (Table V). The univariate analysis identified that the menopausal status, tumor size, histological and TNM stages and lymph node metastasis were significantly associated with the OS and DFS of breast 
a

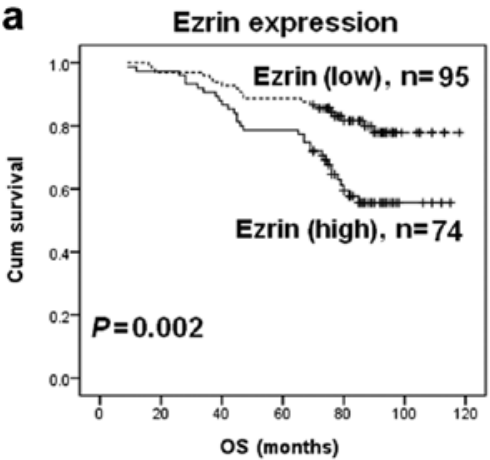

b

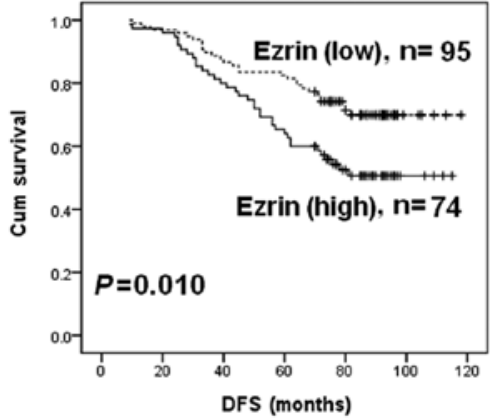

C E-cadherin expression

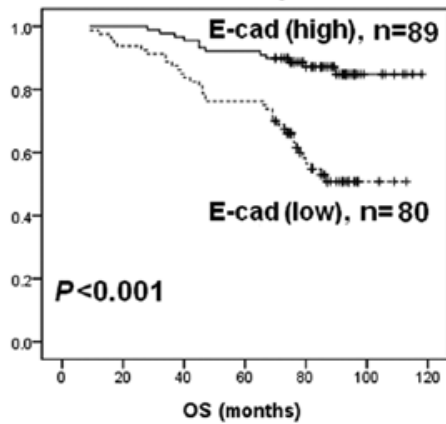

d E-cadherin expression

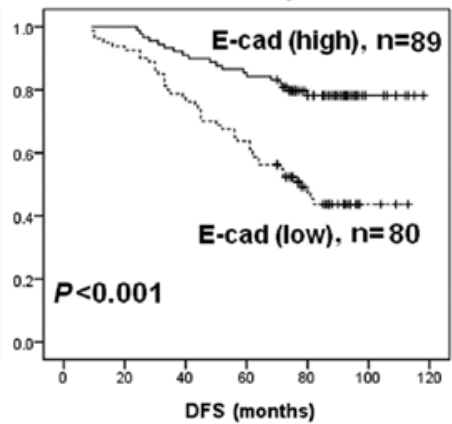

e Localization of E-cadherin

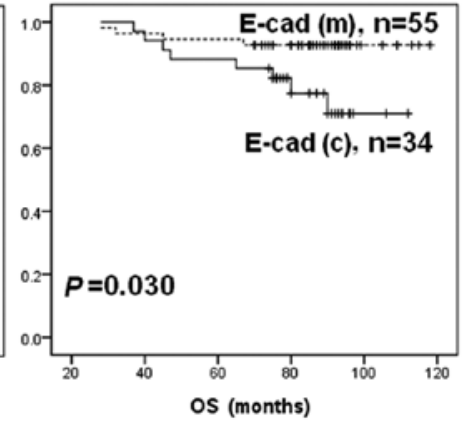

f Localization of E-cadherin

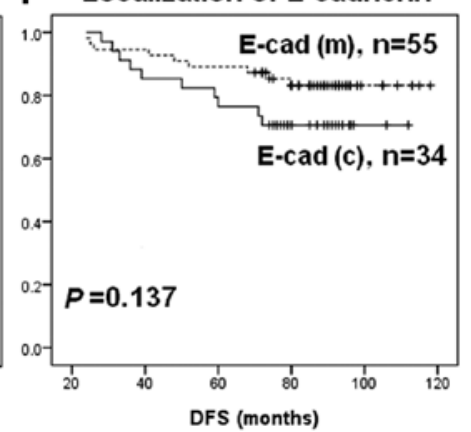

Figure 4. Kaplan-Meier survival analysis of ezrin and E-cadherin expression in breast cancer patients. (a and b) Survival curves show the association between ezrin expression and (a) overall survival (OS) or (b) disease-free survival (DFS) in 169 breast cancer patients. (c and d) Survival curves show the association between E-cadherin expression and (c) overall survival (OS) or (d) disease-free survival (DFS) in 169 breast cancer patients. (e and f) Survival curves show the association between the subcellular expression of E-cadherin and (e) OS or (f) DFS in 89 breast cancer patients.

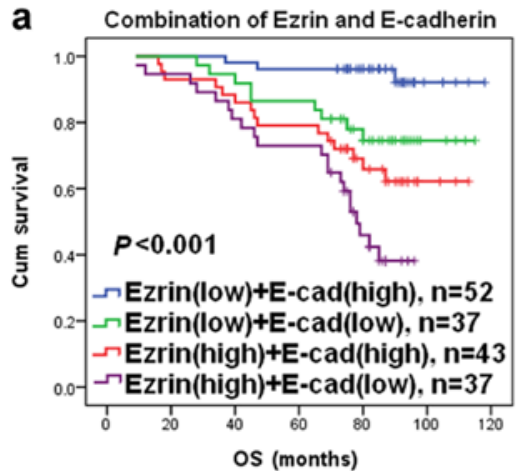

b Combination of Ezrin and E-cadherin

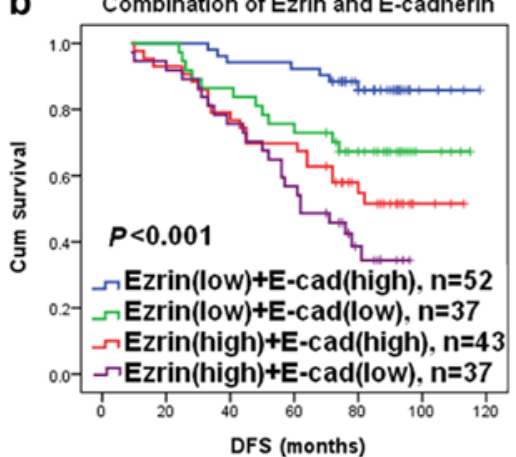

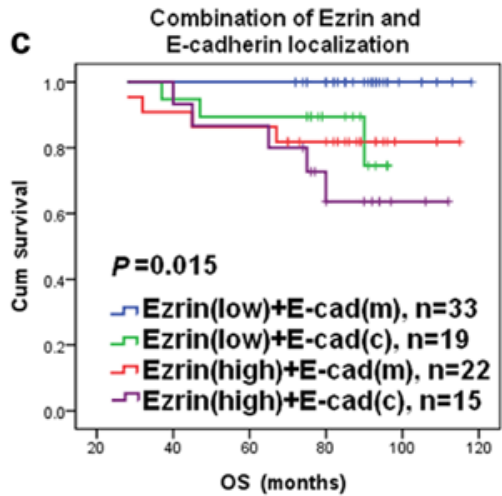

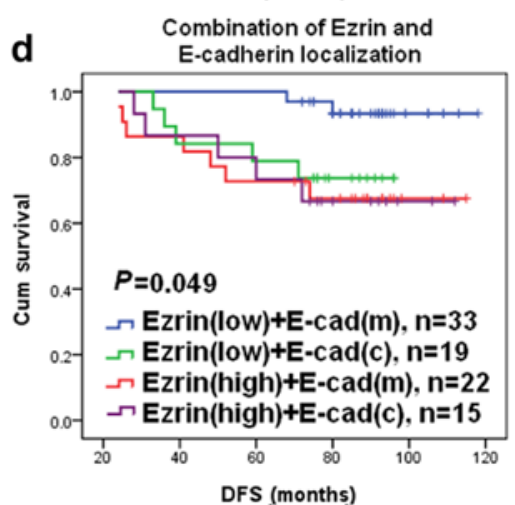

Figure 5. Kaplan-Meier survival analysis of the combined expression of ezrin and E-cadherin in breast cancer patients. (a and b) Survival curves show the association between the combined expression of ezrin and E-cadherin and (a) overall survival (OS) or (b) disease-free survival (DFS) in 169 breast cancer patients. (c and d) Survival curves show the association of the combined expression of ezrin and E-cad(m) or E-cad(c) with (c) OS or (d) DFS in 89 breast cancer patients.

cancer patients (Table V). In addition, ezrin(high) expression was significantly associated with shorter OS and DFS of breast cancer patients. E-cad(c) expression was significantly associated with shorter OS of breast cancer patients (Table V). 
Table V. Univariate Cox regression analysis of the association between clinicopathological data and overall survival (OS) and disease-free survival (DFS) in breast cancer patients.

\begin{tabular}{|c|c|c|c|c|c|c|c|}
\hline \multirow[b]{2}{*}{ Characteristics } & \multirow[b]{2}{*}{$\begin{array}{c}\text { Total } \\
\mathrm{n}\end{array}$} & \multicolumn{3}{|c|}{ OS } & \multicolumn{3}{|c|}{ DFS } \\
\hline & & $\begin{array}{c}\text { Events } \\
\mathrm{n}(\%)\end{array}$ & RR $(95 \% \mathrm{CI})$ & $\mathrm{P}$ & $\begin{array}{c}\text { Events } \\
\mathrm{n}(\%)\end{array}$ & $\mathrm{RR}(95 \% \mathrm{CI})$ & $\mathrm{P}$ \\
\hline Age (years) & & & & 0.104 & & & 0.081 \\
\hline$\leq 50$ & 86 & $20(23.3)$ & 1 (reference) & & $26(30.2)$ & 1 (reference) & \\
\hline$>50$ & 83 & $28(33.7)$ & $1.609(0.906-2.857)$ & & $36(43.4)$ & $1.568(0.946-2.597)$ & \\
\hline Menopaused status & & & & 0.034 & & & 0.029 \\
\hline Premenopause & 86 & $19(22.1)$ & 1 (reference) & & $25(29.1)$ & 1 (reference) & \\
\hline Postmenopause & 78 & $29(37.2)$ & $1.870(1.048-3.335)$ & & $35(44.9)$ & $1.775(1.062-2.966)$ & \\
\hline Tumor size (cm) & & & & $<0.001$ & & & $<0.001$ \\
\hline$\leq 2.0$ & 77 & $11(14.3)$ & 1 (reference) & & $20(26.0)$ & 1 (reference) & \\
\hline$>2.0$ & 60 & $33(55.0)$ & $5.141(2.593-10.19)$ & & $38(63.3)$ & $3.413(1.979-5.885)$ & \\
\hline Tumor type & & & & 0.658 & & & 0.863 \\
\hline Ductal & 130 & $37(28.5)$ & 1 (reference) & & $49(37.7)$ & 1 (reference) & \\
\hline Lobular & 19 & $6(31.6)$ & $1.215(0.513-2.880)$ & & $7(36.8)$ & $1.072(0.486-2.368)$ & \\
\hline Histological grade & & & & $<0.001$ & & & $<0.001$ \\
\hline I & 80 & $7(8.8)$ & 1 (reference) & & $14(17.5)$ & 1 (reference) & \\
\hline II-III & 83 & $41(49.4)$ & $7.327(3.267-16.39)$ & & $48(57.8)$ & $4.393(2.417-7.984)$ & \\
\hline TNM stage & & & & $<0.001$ & & & $<0.001$ \\
\hline I-II & 94 & $23(24.5)$ & 1 (reference) & & $31(33.0)$ & 1 (reference) & \\
\hline III & 23 & $18(78.3)$ & $5.842(3.120-10.94)$ & & $19(82.6)$ & $4.997(2.782-8.974)$ & \\
\hline Lymph node metastasis & & & & $<0.001$ & & & $<0.001$ \\
\hline No & 96 & $12(12.5)$ & 1 (reference) & & $17(17.7)$ & 1 (reference) & \\
\hline Yes & 65 & $33(50.8)$ & $5.134(2.647-9.958)$ & & $42(64.6)$ & $5.032(2.856-8.867)$ & \\
\hline Ezrin expression & & & & 0.003 & & & 0.011 \\
\hline Low & 95 & $18(18.9)$ & 1 (reference) & & $27(28.4)$ & 1 (reference) & \\
\hline High & 74 & $30(40.5)$ & $2.441(1.359-4.382)$ & & $35(47.3)$ & $1.914(1.158-3.164)$ & \\
\hline E-cadherin localization & & & & 0.042 & & & 0.145 \\
\hline E-cad(m) & 55 & $4(7.3)$ & 1 (reference) & & $9(16.4)$ & 1 (reference) & \\
\hline E-cad(c) & 34 & $8(23.5)$ & $1.869(1.024-3.410)$ & & $10(29.4)$ & $1.399(0.891-2.197)$ & \\
\hline
\end{tabular}

RR, relative risk; 95\% CI, 95\% confidence interval; E-cad(m), membrane expression of E-cadherin; E-cad(c), cytoplasmic expression of E-cadherin. Bold, statistically significant.

Table VI. Multivariate Cox regression analysis of the association between clinicopathological data and overall survival (OS) and disease-free survival (DFS) in breast cancer patients.

\begin{tabular}{|c|c|c|c|c|}
\hline \multirow[b]{2}{*}{ Characteristics } & \multicolumn{2}{|l|}{ OS } & \multicolumn{2}{|l|}{ DFS } \\
\hline & $\mathrm{RR}(95 \% \mathrm{CI})$ & $\mathrm{P}$ & $\mathrm{RR}(95 \% \mathrm{CI})$ & $\mathrm{P}$ \\
\hline Age (years) $(>50 / \leq 50)$ & $0.116(0.003-4.857)$ & 0.258 & $0.172(0.007-4.268)$ & 0.283 \\
\hline Menopaused status (post/pre) & $1.074(0.034-33.79)$ & 0.967 & $0.442(0.017-11.65)$ & 0.625 \\
\hline Tumor size $(\mathrm{cm})(>2.0 / \leq 2.0)$ & $7.990(0.522-122.4)$ & 0.136 & $9.054(0.517-158.6)$ & 0.132 \\
\hline Histological grade (II-III/I) & $1.481(0.119-18.42)$ & 0.760 & $0.785(0.093-6.660)$ & 0.824 \\
\hline TNM stage (III/I-II) & $0.514(0.060-4.422)$ & 0.544 & $1.357(0.233-7.903)$ & 0.734 \\
\hline Lymph node metastasis (yes/no) & $51.43(2.283-1158)$ & 0.013 & $122.2(6.819-2188)$ & 0.001 \\
\hline Ezrin (high/low) & $12.17(1.405-105.4)$ & $\mathbf{0 . 0 2 3}$ & $5.935(0.945-36.94)$ & 0.056 \\
\hline E-cadherin localization (c/m) & $0.522(0.137-1.982)$ & 0.340 & $0.554(0.150-2.048)$ & 0.376 \\
\hline
\end{tabular}

$\mathrm{n}=42$. Bold, statistically significant. 
Furthermore, multivariate Cox regression analysis found that lymph node metastasis was an independent prognostic factor for shorter OS and DFS in breast cancer patients $(\mathrm{p}<0.05$, Table VI). Ezrin(high) expression was an independent prognostic factor for shorter OS in breast cancer patients $(\mathrm{p}<0.05$, Table VI).

\section{Discussion}

In the present study, we examined the expression of ezrin and E-cad in 275 breast cancer and 80 control patients with benign hyperplasia. We found that the expression of ezrin was increased in breast cancer samples compared with the control samples. Our finding that ezrin was overexpressed in breast cancer is consistent with findings of previous studies showing ezrin overexpression in tumors including breast cancer $(12,13)$. In addition, we found that ezrin was mainly expressed in the cytoplasm of breast cancer cells. Consistent with this finding, several studies have shown that ezrin is mainly expressed in the cytoplasm of cells from breast cancer patients $(12,27)$. By contrast, we found that E-cad expression was decreased in breast cancer samples compared with the control samples, and E-cad was expressed in the membrane and cytoplasm of cells from breast cancer patients. Our findings agree with those of a previous study showing that E-cad is downregulated in breast cancer and is associated with breast cancer metastasis (23). However, it is unclear whether E-cad downregulation is associated with ezrin overexpression in breast cancer. In the present study, we found that the expression of ezrin was negatively correlated with the expression of E-cad in a subpopulation of breast cancer with ezrin(high) and E-cad(low) expression, suggesting that ezrin contributes to the downregulation of E-cad in this population of breast cancer. This finding was supported by a previous report showing that ezrin knockdown upregulates the expression of E-cad in breast cancer cell lines (15). In addition, the results showed that in breast cancer with E-cad(high) expression, E-cad(c) expression occurred significantly more frequently in ezrin(high) breast cancer than in ezrin(low) breast cancer, and the expression level of ezrin was significantly higher in E-cad(c) breast cancer than in E-cad(m). These findings suggest that ezrin promotes the translocation of E-cad from the membrane to the cytoplasm in breast cancer. This idea was supported by a previous report showing that the active form of ezrin T567D increased the accumulation of E-cad in the cytoplasm accompanied with a decrease in the membrane expression of E-cad (28). Taken together, result of the present study suggest that ezrin regulates the expression of E-cad in breast cancer.

In the present study, we examined the association of the expression of ezrin with the clinicopathological characteristics of breast cancer patients. We found that ezrin(high) expression was associated with lymph node metastasis in breast cancer patients. Consistent with our finding, several studies have shown that ezrin overexpression is associated with lymph-node metastasis in breast cancer $(12,13)$. These findings suggest that ezrin overexpression promotes breast cancer metastasis. The role of ezrin overexpression in breast cancer metastasis is also supported by studies showing that the overexpression of ezrin promotes breast cancer cell metastasis and the knockdown of ezrin by siRNA reverses the metastatic behavior of breast cancer cells $(14,15)$. Furthermore, in the present study, we found that ezrin(high) expression was associated with shorter OS and DFS in breast cancer patients. The multivariate cox regression analysis revealed that ezrin(high) expression was an independent prognostic factor for shorter OS and DFS in breast cancer patients. Consistent with our findings, Gschwantler-Kaulich et al reported that an increased ezrin expression was an independent predictor of invasive breast cancer (12).

In the present study, we also found that E-cad(low) expression was associated with lymph-node metastasis in breast cancer patients, consistent with previous studies showing that the downregulation of E-cad is associated with the invasiveness and metastasis of breast cancer $(23,29,30)$. E-cad(low) expression was associated with shorter OS and DFS in breast cancer patients, suggesting that a reduced expression of E-cad is associated with greater invasiveness and tumor malignancy. Similarly, several findings have shown that reduced or loss of E-cad expression is associated with shorter OS and DFS in breast cancer patients $(22,29,31)$. In addition, E-cad(c) expression was more associated with lymph-node metastasis compared with E-cad(m) expression in the present study, suggesting that the cytoplasmic expression of E-cad is important for breast cancer metastasis. Kowalski et al reported that E-cad was only accumulated in the cytoplasm of invasive lobular carcinoma that developed distant metastases (32). The role of the cytoplasmic expression of E-cad in tumor metastasis is also supported by studies showing that cytoplasmic E-cad expression is associated with the cell invasiveness of lung cancer (33) and breast cancer (34) cell lines. Furthermore, we found that E-cad(c) expression was associated with shorter OS in breast cancer patients, further confirming that the cytoplasmic expression of E-cad is associated with breast cancer malignancy.

We investigated the association between the combined expression of ezrin and E-cad and lymph node metastasis in breast cancer patients. We found that ezrin(high) and E-cad(low) expression was more associated with lymph node metastasis compared with other combined expressions of ezrin and E-cad. Since we found that ezrin expression was negatively correlated with the expression of E-cad in breast cancer with ezrin(high) and E-cad(low) expression, ezrin promotes tumor metastasis via the downregulation of E-cad. This idea is supported by a previous finding that ezrin silencing increased the expression of E-cad, and reversed the metastatic behavior of human breast cancer cells (15). Furthermore, we found that ezrin(high) and E-cad(low) expression was associated with shorter OS and DFS in breast cancer patients, suggesting that ezrin and E-cad may cooperatively regulate tumor metastasis, and thus lead to a poor outcome of breast cancer patients. In addition, we found that ezrin(high) and E-cad(c) expression were more associated with lymph node metastasis and shorter OS and DFS in breast cancer patients. It has been reported that ezrin promotes the translocation of E-cad from the membrane to the cytoplasm via activation of the GTPas Racl (28). It is possible that ezrin regulates the translocation of E-cad in a similar manner, thus promoting the invasion and metastasis of breast cancer.

In summary, we examined the expression of ezrin and E-cad in breast cancer, and analyzed the correlation of ezrin 
and E-cad expression alone and in combination with the clinicopathological characteristics and prognosis of breast cancer patients. We found that ezrin(high), E-cad(low) or E-cad(c) expression was associated with lymph node metastasis and poor prognosis in breast cancer patients. A combined expression of ezrin(high) and E-cad(low) or ezrin(high) and E-cad(c) was more associated with lymph node metastasis and poor prognosis. The present study therefore suggests that ezrin promotes breast cancer metastasis via the regulation of E-cad expression.

\section{Acknowledgements}

This study was supported by grants from the National Natural Science Foundation of the People's Republic of China (no. 81373427), and the Program for Liaoning Innovative Research Team in University, LNIRT (LT2014016).

\section{References}

1. Lu J, Steeg PS, Price JE, Krishnamurthy S, Mani SA, Reuben J, Cristofanilli M, Dontu G, Bidaut L, Valero V, et al: Breast cancer metastasis: Challenges and opportunities. Cancer Res 69: 4951-4953, 2009.

2. O'Shaughnessy J: Extending survival with chemotherapy in metastatic breast cancer. Oncologist 10 (Suppl 3): S20-S29, 2005

3. Friedl $\mathrm{P}$ and Wolf $\mathrm{K}$ : Tumour-cell invasion and migration: Diversity and escape mechanisms. Nat Rev Cancer 3: 362-374, 2003.

4. Tsukita S and Yonemura S: ERM (ezrin/radixin/moesin) family: From cytoskeleton to signal transduction. Curr Opin Cell Biol 9: 70-75, 1997.

5. Nestl A, Von Stein OD, Zatloukal K, Thies WG, Herrlich P, Hofmann M and Sleeman JP: Gene expression patterns associated with the metastatic phenotype in rodent and human tumors. Cancer Res 61: 1569-1577, 2001.

6. Yu H, Zhang Y, Ye L and Jiang WG: The FERM family proteins in cancer invasion and metastasis. Front Biosci 16: 1536-1550, 2011.

7. Yu Y, Khan J, Khanna C, Helman L, Meltzer PS and Merlino G: Expression profiling identifies the cytoskeletal organizer ezrin and the developmental homeoprotein Six-1 as key metastatic regulators. Nat Med 10: 175-181, 2004.

8. Khanna C, Wan X, Bose S, Cassaday R, Olomu O, Mendoza A, Yeung C, Gorlick R, Hewitt SM and Helman LJ: The membranecytoskeleton linker ezrin is necessary for osteosarcoma metastasis. Nat Med 10: 182-186, 2004.

9. Meng Y, Lu Z, Yu S, Zhang Q, Ma Y and Chen J: Ezrin promotes invasion and metastasis of pancreatic cancer cells. J Transl Med 8: 61, 2010.

10. Kang YK, Hong SW, Lee H and Kim WH: Prognostic implications of ezrin expression in human hepatocellular carcinoma. Mol Carcinog 49: 798-804, 2010.

11. Saito S, Yamamoto H, Mukaisho K, Sato S, Higo T, Hattori T, Yamamoto G and Sugihara H: Mechanisms underlying cancer progression caused by ezrin overexpression in tongue squamous cell carcinoma. PLoS One 8: e54881, 2013.

12. Gschwantler-Kaulich D, Natter C, Steurer S, Walter I, Thomas A, Salama $M$ and Singer CF: Increase in ezrin expression from benign to malignant breast tumours. Cell Oncol 36: 485-491, 2013.

13. Halon A, Donizy P, Surowiak P and Matkowski R: ERM/Rho protein expression in ductal breast cancer: A 15 year follow-up. Cell Oncol 36: 181-190, 2013.

14. Elliott BE, Meens JA, SenGupta SK, Louvard D and Arpin M: The membrane cytoskeletal crosslinker ezrin is required for metastasis of breast carcinoma cells. Breast Cancer Res 7: R365-R373, 2005.

15. Li Q, Wu M, Wang H, Xu G, Zhu T, Zhang Y, Liu P, Song A, Gang C, Han Z, et al: Ezrin silencing by small hairpin RNA reverses metastatic behaviors of human breast cancer cells. Cancer Lett 261: 55-63, 2008.
16. Yang $\mathbf{J}$ and Weinberg RA: Epithelial-mesenchymal transition: At the crossroads of development and tumor metastasis. Dev Cell 14: 818-829, 2008.

17. Jeanes A, Gottardi CJ and Yap AS: Cadherins and cancer: How does cadherin dysfunction promote tumor progression? Oncogene 27: 6920-6929, 2008.

18. Foschini MP, Leonardi E, Eusebi LH, Farnedi A, Poli T, Tarsitano A, Cocchi R, Marchetti C, Gentile L, Sesenna E, et al: Podoplanin and E-cadherin expression in preoperative incisional biopsies of oral squamous cell carcinoma is related to lymph node metastases. Int J Surg Pathol 21: 133-141, 2013.

19. Wang C, Jiang K, Kang X, Gao D, Sun C, Li Y, Sun L, Zhang S, Liu X, Wu W, et al: Tumor-derived secretory clusterin induces epithelial-mesenchymal transition and facilitates hepatocellular carcinoma metastasis. Int J Biochem Cell Biol 44: 2308-2320, 2012.

20. Wang WS, Yu SL, Yang XS, Chang SD and Hou JQ: Expression and significance of twist and E-cadherin in ovarian cancer tissues. Asian Pac J Cancer Prev 14: 669-672, 2013.

21. Stanczak A, Stec R, Bodnar L, Olszewski W, Cichowicz M, Kozlowski W, Szczylik C, Pietrucha T, Wieczorek M and Lamparska-Przybysz M: Prognostic significance of Wnt-1, $\beta$-catenin and E-cadherin expression in advanced colorectal carcinoma. Pathol Oncol Res 17: 955-963, 2011.

22. Rakha EA, Patel A, Powe DG, Benhasouna A, Green AR, Lambros MB, Reis-Filho JS and Ellis IO: Clinical and biological significance of E-cadherin protein expression in invasive lobular carcinoma of the breast. Am J Surg Pathol 34: 1472-1479, 2010.

23. Oka H, Shiozaki H, Kobayashi K, Inoue M, Tahara H, Kobayashi T, Takatsuka Y, Matsuyoshi N, Hirano S, Takeichi M, et al: Expression of E-cadherin cell adhesion molecules in human breast cancer tissues and its relationship to metastasis. Cancer Res 53: 1696-1701, 1993.

24. Chen MJ, Gao XJ, Xu LN, Liu TF, Liu XH and Liu LX: Ezrin is required for epithelial-mesenchymal transition induced by TGF- $\beta 1$ in A549 cells. Int J Oncol 45: 1515-1522, 2014.

25. Fang Y, Wei J, Cao J, Zhao H, Liao B, Qiu S, Wang D, Luo J and Chen W: Protein expression of ZEB2 in renal cell carcinoma and its prognostic significance in patient survival. PLoS One 8: e62558, 2013.

26. Zhu W, Cai MY, Tong ZT, Dong SS, Mai SJ, Liao YJ, Bian XW, Lin MC, Kung HF, Zeng YX, et al: Overexpression of EIF5A2 promotes colorectal carcinoma cell aggressiveness by upregulating MTA1 through C-myc to induce epithelial-mesenchymaltransition. Gut 61: 562-575, 2012.

27. Sarrió D, Rodríguez-Pinilla SM, Dotor A, Calero F, Hardisson D and Palacios J: Abnormal ezrin localization is associated with clinicopathological features in invasive breast carcinomas. Breast Cancer Res Treat 98: 71-79, 2006.

28. Pujuguet P, Del Maestro L, Gautreau A, Louvard D and Arpin M: Ezrin regulates E-cadherin-dependent adherens junction assembly through Rac1 activation. Mol Biol Cell 14: 2181-2191, 2003.

29. Siitonen SM, Kononen JT, Helin HJ, Rantala IS, Holli KA and Isola JJ: Reduced E-cadherin expression is associated with invasiveness and unfavorable prognosis in breast cancer. Am J Clin Pathol 105: 394-402, 1996.

30. Yoshida R, Kimura N, Harada Y and Ohuchi N: The loss of E-cadherin, $\alpha$ - and $\beta$-catenin expression is associated with metastasis and poor prognosis in invasive breast cancer. Int $\mathbf{J}$ Oncol 18: 513-520, 2001.

31. Brzozowska A, Sodolski T, Duma D, Mazurkiewicz T and Mazurkiewicz M: Evaluation of prognostic parameters of E-cadherin status in breast cancer treatment. Ann Agric Environ Med 19: 541-546, 2012.

32. Kowalski PJ, Rubin MA and Kleer CG: E-cadherin expression in primary carcinomas of the breast and its distant metastases. Breast Cancer Res 5: R217-R222, 2003.

33. Zhang Y, Zhao Y, Jiang G, Zhang X, Zhao H, Wu J, Xu K and Wang E: Impact of p120-catenin isoforms $1 \mathrm{~A}$ and $3 \mathrm{~A}$ on epithelial mesenchymal transition of lung cancer cells expressing E-cadherin in different subcellular locations. PLoS One 9: e88064, 2014.

34. Lapyckyj L, Castillo LF, Matos ML, Gabrielli NM, Lüthy IA and Vazquez-Levin MH: Expression analysis of epithelial cadherin and related proteins in IBH-6 and IBH-4 human breast cancer cell lines. J Cell Physiol 222: 596-605, 2010. 